\title{
Atenção auditiva sustentada em crianças com transtorno de déficit de atenção e hiperatividade: o efeito da medicação
}

\author{
Sustained auditory attention in children with attention deficit \\ hyperactivity disorder: the effects of medication
}

\author{
Núbia Machado Michels ${ }^{1}$ (D), Cláudia Maria de Lorenzo ${ }^{2}$ (D), João Carlos Xikota ${ }^{2,3}$ (D), Danúbia Hillesheim ${ }^{4}$ (D), \\ Evandra Castro Donatti ${ }^{2}$ (D), Maria Madalena Canina Pinheiro' ${ }^{10}$
}

\section{RESUMO}

Objetivo: Comparar a habilidade atencional auditiva em crianças portadoras de transtorno de déficit de atenção e hiperatividade/impulsividade (TDAH) antes e após o uso da medicação psicoestimulante. Métodos: Participaram do estudo oito crianças do gênero masculino, com TDAH, entre 8 e 10 anos de idade. Todas faziam uso de medicamentos psicoestimulantes. Para avaliar o comportamento auditivo, foi aplicado o questionário Scale of Auditory Behaviors (SAB) e para identificar possíveis sintomas de desatenção, o Swanson, Nolan e Pelham (SNAP-IV). A habilidade auditiva atencional foi avaliada pelo Teste de Habilidade de Atenção Auditiva Sustentada (THAAS) pré e pós-uso do medicamento psicoestimulante. Neste teste, foram analisados os erros de desatenção, de impulsividade e de decréscimo de vigilância. A análise dos dados foi feita com base nos testes Wilcoxon e Correlação de Spearman. Resultados: O questionário SAB mostrou-se alterado na maior parte das crianças, as quais também apresentaram sinais de desatenção e/ou impulsividade no questionário SNAP-IV. No THASS, observou-se diferença nos quesitos desatenção $(\mathrm{p}=0,017)$ e pontuação total de erros pré e pós-medicação $(\mathrm{p}=0,025)$. Conclusão: Houve melhora no desempenho do teste que avaliou a habilidade atencional auditiva após o uso da medicação psicoestimulante, com destaque para o item desatenção.

Palavras-chave: Atenção; Percepção auditiva; Testes auditivos; Transtorno do déficit de atenção e hiperatividade; Criança

\begin{abstract}
Purpose: To compare the auditory attention ability in children with Attention Deficit Hyperactivity Disorder (ADHD) before and after the use of psychostimulant medication. Methods: Eight male children with ADHD participated in the study, between eight and ten years old. All participants used psychostimulant drugs. To assess auditory behavior, the Scale of Auditory Behaviors (SAB) and SNAP-IV questionnaires were applied to identify possible symptoms of inattention and/or impulsivity. To assess auditory attention ability, the Sustained Auditory Attention Ability Test (SAAAT) was applied before and after the use of the psychostimulant medication. In this test, the errors of inattention, impulsiveness and decreased vigilance were analyzed. For data analysis, the Wilcoxon and Spearman correlation tests were used. Results: The SAB questionnaire showed changes in most children, who also showed signs of inattention and/or impulsivity in the SNAP-IV questionnaire. In the SAAAT, a difference was observed in the item inattention $(\mathrm{p}=0.017)$ and in total score of errors before and after medication $(\mathrm{p}=0.025)$. Conclusion: There was an improvement in the test performance assessing the auditory attention ability after the use of psychostimulant medication, with emphasis on the item inattention.
\end{abstract}

Keywords: Attention; Auditory perception; Hearing tests; Attention deficit hyperactivity disorder; Child

\footnotetext{
Trabalho realizado no Curso de Fonoaudiologia, Universidade Federal de Santa Catarina - UFSC - Florianópolis (SC), Brasil.

${ }^{1}$ Universidade Federal de Santa Catarina - UFSC - Florianópolis (SC), Brasil.

${ }^{2}$ Núcleo Desenvolver - UASCA, Hospital Universitário Professor Polydoro Ernani de São Thiago/ Empresa Brasileira de Serviços Hospitalares-EBSERH - Florianópolis (SC), Brasil.

${ }^{3}$ Departamento de Pediatria, Universidade Federal de Santa Catarina - UFSC - Florianópolis (SC), Brasil.

${ }^{4}$ Programa de Pós-graduação em Saúde Coletiva, Universidade Federal de Santa Catarina - UFSC - Florianópolis (SC), Brasil.

Conflito de interesses: Não.

Contribuição dos autores: NMM participou da concepção e delineamento do estudo, foi responsável pela coleta e análise dos dados e escrita do manuscrito; CML, JCX e ECD auxiliaram na coleta e na análise dos dados; DH contribuiu com a análise dos dados e escrita do manuscrito; MMCP participou da concepção e delineamento do estudo, foi responsável pela orientação do estudo, análise dos dados e escrita do manuscrito.

Financiamento: Nada a declarar.
}

Autor correspondente: Maria Madalena Canina Pinheiro. E-mail: madacanina@gmail.com

Recebido: Julho 14, 2020; Aceito: Novembro 11, 2020 


\section{INTRODUÇÃO}

O mecanismo atencional é extremamente essencial na cognição, pois é um processo neuropsicológico básico das funções executivas ${ }^{(1)}$. Uma de suas finalidades é permitir que o sujeito perceba e analise estímulos auditivos distintos a qualquer momento $^{(2)}$, contribuindo, de forma decisiva, para a aquisição de aspectos acústicos e fonéticos dos padrões linguísticos, fundamentais para a evolução das habilidades comunicativas e de aprendizagem ${ }^{(3)}$.

Para a informação ser processada, existem diferentes tipos de atenção, dentre elas, a atenção sustentada, que permite ao indivíduo começar e terminar uma tarefa em um determinado período de tempo ${ }^{(4,5)}$. A alteração nesta habilidade faz com que o indivíduo perca ou não registre as informações em sua memória, tornando-o desatento e distraído ${ }^{(6)}$.

Os deficit atencionais auditivos podem ser encontrados em outros diagnósticos, como no distúrbio de linguagem, no transtorno de deficit de atenção e hiperatividade (TDAH) e no transtorno do processamento auditivo central (TPAC) ${ }^{(7)}$. O TPAC corresponde a um deficit na eficácia e na efetividade com que o sistema nervoso central (SNC) processa e interpreta os sons verbais ou não verbais ${ }^{(8)}$. Além disso, o TPAC deve ser compreendido como um transtorno que pode ser isolado ou associado a outras comorbidades ${ }^{(7)}$.

Clínicos e pesquisadores publicaram, em 2017, o Consenso Europeu sobre o TPAC ${ }^{(9)}$, apontando o caminho ideal para o diagnóstico e para o manejo adequado dos casos. Segundo o documento, na presença do TDAH, deve ser indicada a avaliação do processamento auditivo central (PAC), especialmente nos casos em que uma terapia apropriada e a contribuição educacional não mostraram resultados esperados ou adequados ${ }^{(9)}$.

O TDAH é definido como um transtorno do neurodesenvolvimento, caracterizado por apresentar níveis frequentes de desatenção, hiperatividade e impulsividade ${ }^{(10)}$. Atualmente, a estreita relação entre TDAH e TPAC tem chamado a atenção de estudiosos, devido à dificuldade enfrentada na realização do diagnóstico diferencial ${ }^{(11-13)}$. É de suma importância que essas manifestações sejam investigadas o mais breve possível, principalmente quando começam a interferir na aprendizagem, causando prejuízos escolares e sociais ${ }^{(12)}$.

$\mathrm{Na}$ literatura, há extensa lista de fatores positivos sobre o uso de medicação para TDAH. Dentre os benefícios, destaca-se a melhora do desempenho em testes especiais do PAC nas crianças, após o uso do medicamento ${ }^{(14)}$.

A escolha por tratamento medicamentoso se sobressai em relação a intervenções sociais, psicológicas e comportamentais. Em todo o mundo, a substância mais utilizada tem sido o metilfenidato, que atua na estimulação do SNC, causando o aumento da atenção e a diminuição da inquietação em crianças hiperativas ${ }^{(14)}$.

Com o intuito de avaliar a atenção auditiva, surgiu, nos últimos anos, o Teste de Habilidade de Atenção Auditiva Sustentada (THAAS), que avalia o nível atencional auditivo durante um período de tempo prolongado ${ }^{(6)}$. No Fórum Diagnóstico Audiológico da Academia Brasileira de Audiologia ${ }^{(15)}$,de 2016, o THAAS foi recomendado para fazer parte do protocolo mínimo de avaliação do PAC de crianças com dificuldades atencionais. Tendo em vista essa orientação e a carência na literatura nacional de trabalhos com o THAAS em indivíduos com TDAH, este estudo buscou evidenciar a importância da sua implantação na bateria de testes do PAC e contribuir para o emprego clínico da avaliação da habilidade atencional auditiva sustentada.

Diante desse contexto, este trabalho teve o objetivo de comparar a habilidade atencional auditiva em crianças portadoras do TDAH antes e após o uso da medicação psicoestimulante.

\section{MÉTODO}

Este é um estudo transversal, descritivo e observacional. A coleta foi realizada de forma primária e a seleção da população, de maneira não probabilística, por conveniência.

A pesquisa foi aprovada pelo Comitê de Ética em Pesquisa da Universidade Federal de Santa Catarina, sob o número de protocolo CAAE 64362517.5.0000.0121 e parecer 3.758.303. Todos os responsáveis pelos menores foram esclarecidos sobre os objetivos da pesquisa e após a sua anuência, assinaram o Termo de Consentimento Livre e Esclarecido (TCLE). A coleta de dados foi realizada no setor de Audiologia da Clínica-Escola de Fonoaudiologia da Universidade Federal de Santa Catarina, no período de março a setembro de 2018.

Participaram deste estudo oito crianças com idade entre 8 e dez anos, todas do gênero masculino, com diagnóstico médico de TDAH. O grupo de crianças com TDAH era diagnosticado e acompanhado pela equipe multidisciplinar do Núcleo Desenvolver do Hospital Universitário Professor Polydoro Ernani de São Thiago da Universidade Federal de Santa Catarina - UFSC, que realizava o diagnóstico previsto no Manual de Diagnóstico e Estatística V (DSM-V).

Foram adotados os seguintes critérios de elegibilidade: crianças com diagnóstico médico de TDAH, que faziam uso de medicamentos psicoestimulantes, com limiares auditivos dentro dos padrões da normalidade, bilateralmente, e que falassem o português brasileiro como primeira língua. Os critérios de exclusão foram: diagnóstico de TDAH com psicometria alterada e/ou presença de outras comorbidades (dislexia, autismo, transtorno opositivo desafiador etc.)

Para caracterizar a população, foi realizada com os responsáveis uma anamnese abordando questões relacionadas à aprendizagem, ao desenvolvimento da audição, da fala e da linguagem, ao sono, às características comportamentais dos indivíduos e à medicalização e acompanhamento com outros profissionais. Posteriormente, foram aplicados os questionários SNAP-IV e Scale of Auditory Behaviors (SAB).

$\mathrm{O}$ questionário SNAP-IV é um instrumento desenvolvido com base nos sintomas do Manual de Diagnóstico e Estatística IV Edição (DSM-IV), da Associação Americana de Psiquiatria. $\mathrm{O}$ teste tem como objetivo rastrear informações a respeito dos possíveis sintomas iniciais presentes nos indivíduos com TDAH, sendo útil para avaliar o primeiro critério diagnóstico do TDAH. O questionário possui 18 itens e apresenta duas partes, a primeira relacionada aos sintomas de desatenção e a segunda, aos sintomas de hiperatividade e impulsividade. Se em uma ou nas duas partes forem marcados seis ou mais itens para os quesitos "bastante e/ ou demais", significa que existem mais sintomas de desatenção e/ou hiperatividade do que o esperado para uma criança ou adolescente sem essas alterações ${ }^{(16)}$. Para análise do questionário SNAP-IV, denominou-se como parte A os itens de desatenção que foram assinalados nas questões de 1 a 9 como "demais e/ou bastante". Da mesma forma, na parte $\mathrm{B}$, nos itens de 10 a 18 , referentes à hiperatividade e à impulsividade. 
$\mathrm{O}$ questionário $\mathrm{SAB}$ é constituído por 12 questões e tem como objetivo avaliar comportamentos relacionados à audição e à atenção. Os responsáveis deveriam respondê-lo, assinalando a opção que indicasse a frequência de um comportamento em seu filho, de acordo com a seguinte escala: (1) sempre, (2) quase sempre, (3) às vezes, (4) esporadicamente e (5) nunca. Após o preenchimento, as respostas foram somadas para obtenção da pontuação total. A pontuação final, quando inferior a 36 , foi considerada sugestiva de risco para a alteração de $P A C^{(17)}$.

Após a realização dos questionários, aplicou-se o THAAS nos pacientes. O THAAS foi desenvolvido com base no teste Auditory Continuous Performance Test (ACPT), com o objetivo de avaliar o comportamento atencional auditivo de crianças. O estímulo do teste é composto por 21 palavras monossilábicas que são repetidas de forma aleatória, formando uma lista de 100 palavras. O estímulo-alvo do teste é a palavra "não", que ocorre 20 vezes de forma aleatória em cada lista. $\mathrm{O}$ indivíduo escutará inicialmente uma lista-treino e, em seguida, seis listas com 100 palavras sem interrupção. Ao ser ouvida a palavraalvo, o indivíduo deveria levantar a mão. O teste, que possui duração média de 11 minutos $^{(6)}$, foi apresentado de forma binaural a $50 \mathrm{dBNS}$, baseado na média tritonal das frequências $500 \mathrm{~Hz}, 1000 \mathrm{~Hz}$ e $2000 \mathrm{~Hz}$. Disponível no site THAAS ${ }^{(18)}$, neste estudo, o teste foi acoplado no audiômetro Madsen Astera ${ }^{2}$. São analisados os seguintes padrões de erro: pontuação total de erros, desatenção, impulsividade e decréscimo da vigilância.

O erro de desatenção é caracterizado quando a criança não levanta a mão em resposta à palavra "não" antes da apresentação da palavra seguinte. Já o erro de impulsividade, ocorre quando a criança levanta a mão para outra palavra, ao invés da palavra "não". A contagem dos números de erros de desatenção e de impulsividade permitiu obter a pontuação total de erros do THAAS. O decréscimo da vigilância é calculado subtraindo o número de respostas corretas da primeira apresentação do número de respostas corretas da última apresentação. Utilizaram-se os critérios de normalidade por faixa etária do THAAS de Feminan e Lemos ${ }^{(6)}$.

Cabe ressaltar que as avaliações dos participantes foram agendadas em dia e horário previamente estabelecidos e tiveram duração média de uma hora e vinte minutos. Todos os indivíduos foram avaliados em dois momentos distintos, com intervalo de uma semana entre um e outro. No primeiro momento, foram realizados todos os testes auditivos especiais sem o efeito da medicação e, no segundo momento, com o uso da medicação, em doses variáveis, de acordo com a prescrição médica.

Todas as avaliações ocorreram no período matutino, para que as chances de fadiga fossem menores. Ressalta-se que, para a reavaliação, foi solicitado aos responsáveis que os pacientes realizassem o exame o mais cedo possível, para não comprometer a rotina de administração do uso da medicação. $\mathrm{Na}$ análise da avaliação simplificada do PAC, foram considerados alterados os indivíduos que tiveram, pelo menos, uma prova alterada e, no THAAS, quando houve alteração em pelo menos um item avaliado.

O equipamento utilizado para a realização das avaliações auditivas foi o audiômetro da marca Otometrics, modelo Madsen Astera $^{2}$, com fone circum-aural Sennheiser HDA 200, em cabina acústica. Este equipamento foi acoplado a um computador, no qual os testes estavam gravados em formato MP3.

Os dados foram computados em planilhas do Microsoft Excel e receberam análise estatística descritiva, por meio da distribuição de frequência das variáveis categóricas e das medidas de tendência central e dispersão das variáveis contínuas. Os dados foram analisados estatisticamente por meio dos programas $M e d C a l c \circledR$, versão 18.10 (MedCalc Software bvba
1993-2018, Bélgica) e IBM SPSS 25. Neste estudo, foram utilizados os testes estatísticos (não paramétricos) Wilcoxon, para comparação de medianas em amostras pareadas e correlação de Spearman. O valor de $\mathrm{p}$ foi considerado significante para valores menores que $5 \%(\mathrm{p}<0,05)$ e foi representado com asterisco sobrescrito $(*)$.

\section{RESULTADOS}

Fizeram parte deste estudo 8 crianças do gênero masculino, sendo 3 com 8 anos, 4 com 9 anos e uma com 10 anos de idade. Quanto à escolaridade, metade $(50,0 \%)$ estava no $4^{\circ}$ ano do ensino fundamental. Todos faziam uso da medicação psicoestimulante, sendo que 7 (87,5\%) utilizavam o metilfenidato e um (12,5\%), lisdexanfetamina. O tempo de uso da medicação variou de 1 a 12 meses (média de tempo de 7,28 meses), com a dose diária variando de 1 a 3 comprimidos. Observou-se que a maior parte das crianças apresentava distúrbios da comunicação humana (Quadro 1).

Para verificar a associação entre os questionários SAB e SNAP-V, foi calculado o coeficiente de correlação de Spearman, porém, não se observou diferença significativa entre eles $(\mathrm{p}=0,275$ para comparação entre a parte B do SNAP-IV e o SAB e p=0,729 para comparação entre a parte A e o SAB) (Tabela 1).

Quadro 1. Escolaridade e dificuldades escolares e de comunicação nos indivíduos com transtorno de deficit de atenção e hiperatividade $(\mathrm{n}=8)$

\begin{tabular}{|c|c|c|}
\hline & $\begin{array}{c}\text { Escolaridade } \\
\text { (anos) }\end{array}$ & $\begin{array}{c}\text { Dificuldade Escolar/ } \\
\text { Comunicação }\end{array}$ \\
\hline Paciente 1 & 3 & - \\
\hline Paciente 2 & 3 & Fala/ Leitura/ Escrita \\
\hline Paciente 3 & 4 & Fala/ Leitura/ Escrita \\
\hline Paciente 4 & 2 & Fala/ Leitura/ Escrita \\
\hline Paciente 5 & 4 & Fala/ Escrita \\
\hline Paciente 6 & 4 & Fala/ Leitura \\
\hline Paciente 7 & 2 & Fala/ Leitura/ Escrita \\
\hline Paciente 8 & 4 & Leitura/Escrita \\
\hline
\end{tabular}

$(-)$ = não apresenta dificuldades escolares e de comunicação

Tabela 1. Desempenho dos indivíduos no questionário Scale of Auditory Behaviors e no Swanson, Nolan e Pelham em dados absolutos $(\mathrm{n}=8)$

\begin{tabular}{ccccc}
\hline & SAB & & \multicolumn{2}{c}{ SNAP-IV } \\
\cline { 2 - 3 } \cline { 4 - 5 } \cline { 4 - 5 } Paciente 1 & N & & A & B \\
Paciente 2 & 39 & & 5 & 0 \\
Paciente 3 & 30 & & 7 & 6 \\
Paciente 4 & 15 & & 7 & 2 \\
Paciente 5 & 17 & & 2 & 8 \\
Paciente 6 & 18 & & 4 & 7 \\
Paciente 7 & 25 & & 6 & 3 \\
Paciente 8 & 22 & & 7 & 6 \\
Média & 25 & & 7 & 2 \\
Desvio Padrão & 7,87 & & 5,62 & 4,25 \\
Mediana & 23,8 & & 1,84 & 2,86 \\
Máxima & 39 & 6,5 & 4,5 \\
Mínimo & 15 & 7 & 8 \\
\hline
\end{tabular}

Legenda: SAB = Scale of Auditory Behaviors; SNAP-IV = Swanson, Nolan e Pelham; $\mathrm{N}$ = numero absoluto; $\mathrm{A}$ = Primeira parte do questionário; $\mathrm{B}=$ Segunda parte do questionário 
Com relação à análise dos tipos de erro do THASS, pôde-se observar que houve diferença no quesito desatenção $(\mathrm{p}=0,017)$ e pontuação total de erros pré e pós-medicação $(\mathrm{p}=0,025)$ (Tabela 2).

A Figura 1 mostra a representação gráfica dos dados do desempenho do THAAS quanto à mediana, à amplitude, à simetria ou assimetria dos dados. Verificou-se que o desempenho de todos os indivíduos melhorou na avaliação com o uso da medicação e que a mediana apresentou menor variação entre os indivíduos testados após a medicação, exceto em relação ao item impulsividade.

\section{DISCUSSÃO}

No TDAH, a dificuldade em sustentar a atenção está entre os sintomas mais predominantes, presente em indivíduos frequentemente rotulados como indisciplinados e distraídos. O comprometimento da manutenção de foco atencional prejudica a capacidade de, simultaneamente, se concentrar em uma tarefa e fazer a inibição de outros estímulos, o que afeta, de forma direta e indireta, o sucesso das atividades. Esses indivíduos podem apresentar grau variado de dificuldades de aprendizagem e comportamento, pior desempenho acadêmico e dano nas interações familiares e sociais. Isso pode acontecer em decorrência da deficiência das habilidades atencionais e de controle inibitório, que comprometerão o sistema fonológico, sintático e pragmático da linguagem ${ }^{(10)}$.

Na presente pesquisa, observou-se que $87,5 \%$ das crianças apresentavam alterações na comunicação oral e na aprendizagem, sendo que muitas necessitavam de auxílio especializado. A avaliação do PAC tem sido cada vez mais recomendada nas crianças que apresentam dificuldades de aprendizagem, em virtude de o exame possibilitar o reconhecimento e o entendimento das dificuldades que o indivíduo possa apresentar ${ }^{(17,19)}$.

O emprego de questionários para auxiliar a análise diagnóstica tem sido frequentemente utilizado por profissionais. A utilização desses instrumentos permite obter informações ricas sobre os pacientes e seus familiares. Verifica-se, na literatura, uma infinidade de questionários que podem ser utilizados para complementar a avaliação do PAC ${ }^{(20)}$.

No presente estudo, a pontuação do questionário SAB foi baixa em sete indivíduos $(87,5 \%)$. Nunes et al. ${ }^{(17)}$ sinalizaram que a pontuação abaixo de 36 sugere risco para TPAC e que,

Tabela 2. Análise exploratória dos tipos de erros do Teste de Habilidade de Atenção Auditiva Sustentada pré e pós-medicação, em valores absolutos $(n=8)$

\begin{tabular}{ccccc}
\hline & Desatenção & Impulsividade & Decréscimo da vigilância & Pontuação total de erros \\
\hline Média Pré & 26,50 & 7,50 & 2,25 & 34 \\
Média Pós & 10,12 & 7,50 & 1,87 & 17,62 \\
Mediana Pré & 20,50 & 6,50 & 1,50 & 25 \\
Mediana Pós & 5,50 & 6,00 & 1,50 & 11,50 \\
Máxima Pré & 66 & 17 & 6 & 83 \\
Máxima Pós & 41 & 20 & 7 & 61 \\
Mínimo Pré & 2 & 3 & 0 & 9 \\
Mínimo Pós & 0 & 2 & 0 & 5 \\
Valor de p & $0,017^{*}$ & 0,865 & 0,786 & $0,025^{*}$ \\
\hline
\end{tabular}

*Teste de Wilcoxon

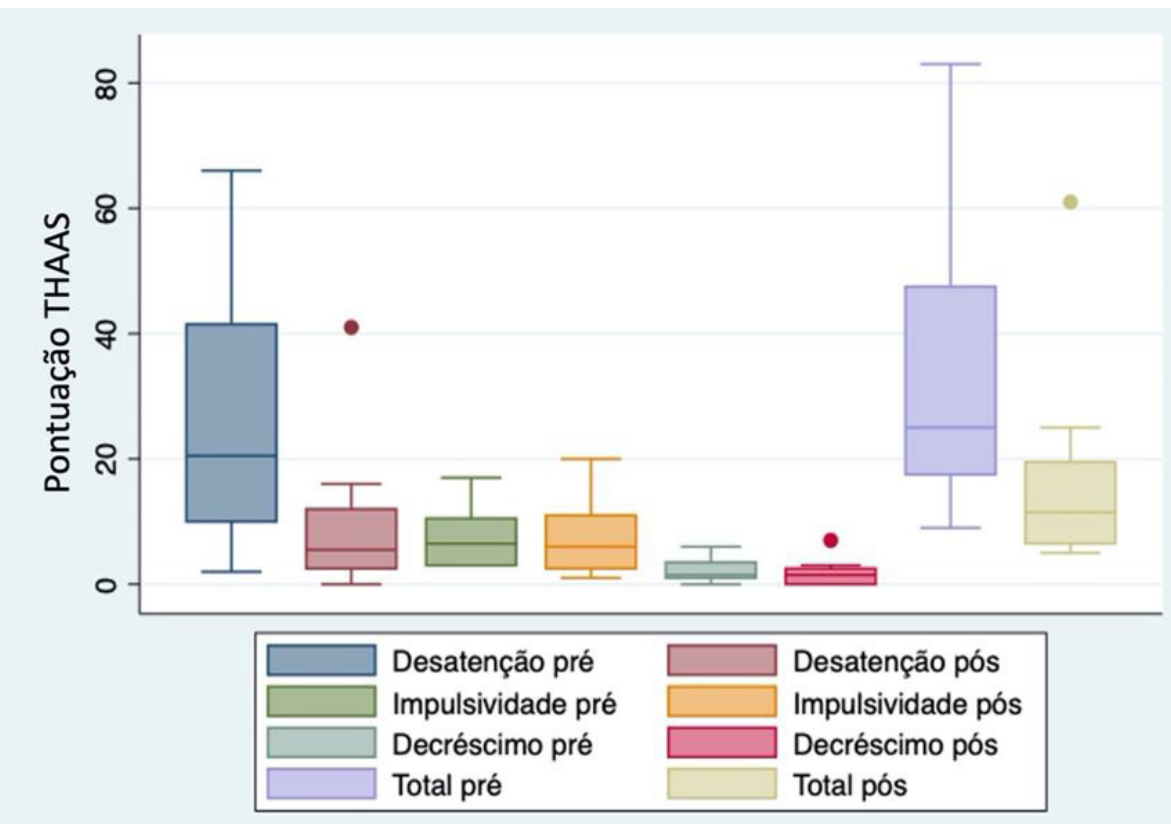

Figura 1. Análise da distribuição dos resultados do Teste de Habilidade de Atenção Auditiva Sustentada pré e pós-medicação Legenda: THAAS = Teste de Habilidade de Atenção Auditiva Sustentada 
nesses casos, as crianças devem ser encaminhadas para realizar a avaliação do PAC. Em relação ao SNAP-IV, foi observado, conforme as alterações das partes A e B, que duas crianças apresentaram TDAH do tipo combinado, duas com predomínio de desatenção e duas com predomínio de hiperatividade/impulsividade, pois obtiveram pontuações iguais ou superiores a 6 nas partes A e/ou B. Constatou-se, também, que todos os indivíduos que tiveram alteração na pontuação do SAB apresentaram alteração em, pelo menos, um dos itens do SNAP-IV, ou seja, 87,5\% das crianças avaliadas.

Miranda et al. ${ }^{(20)}$ demonstraram que o questionário SAB é um importante recurso para triagem, permitindo identificar inabilidades auditivas precocemente e também medir a efetividade das terapias, sendo indicado para o uso rotineiro nas clínicas. Além disso, o estudo mostrou que os resultados do SAB possuem grande correlação com os testes comportamentais, como o TDD. Outro estudo ${ }^{(21)}$ também citou a utilização do SNAP-IV como forma de rastreio, recomendando sua utilização por profissionais da Educação, com o objetivo de auxiliar na identificação de possíveis sintomas relacionados ao TDAH no ambiente escolar.

Desta forma, a utilização de questionários, na anamnese, que avaliem a habilidade atencional em sujeitos com sinais de desatenção é pertinente no contexto clínico e proporciona o adequado manejo do paciente, já que as possíveis alterações em testes do PAC podem estar vinculadas a outros deficit, como os da atenção. A utilização desses instrumentos pode contribuir para o desenvolvimento de estratégias, no sentido de melhorar as habilidades auditivas ou atencionais, e também para o monitoramento dessas inabilidades ${ }^{(16,17,21)}$.

Vale ressaltar que os questionários são importantes ferramentas para auxiliar o diagnóstico, porém, no caso de conclusão de diagnóstico do TDAH, o DMS-V atribui outros quatro critérios para a sua conclusão final. Logo, a investigação do transtorno não pode se dar exclusivamente por meio de um questionário, mas por diferentes testes e procedimentos que tornem o diagnóstico fidedigno ${ }^{(21)}$.

A literatura mostra que após o uso de medicamento psicoestimulante há melhora nos testes comportamentais do PAC. Os dados da atual pesquisa concordam com o estudo de Cavadas et al. ${ }^{(12)}$, no qual se investigou o efeito do uso do medicamento metilfenidato no desempenho de crianças e adolescentes com TDAH nos testes comportamentais padronizados do PAC. Os resultados revelaram melhora no desempenho nos testes após a utilização do medicamento. Os autores referiram que as alterações do PAC nesses indivíduos seriam decorrentes de uma alteração secundária à desatenção, como a dificuldade de aprendizagem.

Uma pesquisa mostrou efeitos positivos do uso do medicamento metilfenidato em adultos com diagnóstico de TDAH. O processamento auditivo temporal desses pacientes, sob efeito da medicação, foi igual ao de adultos sem deficit de atenção. Os autores relataram que, devido à associação entre processamento temporal auditivo e habilidades linguísticas, o efeito benéfico do metilfenidato no indivíduo adulto pode ter resultados positivos em seu desempenho acadêmico ${ }^{(22)}$.

Outro estudo ${ }^{(11)}$ verificou que não houve diferença significativa entre uma avaliação e outra, nos testes do PAC. Desta forma, o estudo concluiu que, por se tratar de um procedimento novo para esses indivíduos, eles teriam mais atenção durante as testagens Os autores aconselharam que as avaliações do PAC fossem realizadas com a medicação, para evitar fatores estressantes para os pacientes e para o avaliador, bem como pequenas pausas entre os testes, remoção de fones e reforço de instruções. O estudo supracitado ${ }^{(1)}$ diverge do presente estudo, uma vez que os autores não encontraram diferença nos resultados entre a utilização da medicação psicoestimulante nas avaliações.

Suspeita-se que o efeito de aprendizagem dos testes possa estar vinculado a divergências dos resultados nas pesquisas que testaram o efeito da medicação. No entanto, não se descarta a hipótese de que os problemas de aprendizagem escolares nos pacientes com TDAH também são fatores que podem influenciar nos resultados do PAC. Além disso, a amostra reduzida também pode ser um fator contribuinte para esses resultados.

Não existe consenso na literatura a respeito de associações e dissociações neurocognitivas entre TDAH e TPAC, o que dificulta o diagnóstico preciso dos deficit que caracterizam um determinado transtorno ou a sua comorbidade ${ }^{(19)}$.

Sabe-se que a atenção sustentada é uma habilidade complexa mediada pelo córtex pré-frontal ${ }^{(3)}$, que permite ao indivíduo começar, manter e terminar uma tarefa em determinado intervalo de tempo ${ }^{(4,5)}$, o que, por sua vez, é fundamental na compreensão da linguagem em tempo real.

No presente estudo, pôde-se observar, nos resultados do THAAS, que houve melhora significativa do desempenho dos participantes após a utilização da medicação, considerando os itens desatenção e pontuação total de erros. Estudo realizado com o THAAS ${ }^{(23)}$, por meio de fones auriculares em campo livre, com crianças normo-ouvintes, verificou o efeito de aprendizagem nos testes, pois a maior parte das crianças melhorou na segunda testagem.

Por ser um instrumento relativamente novo, a repercussão do THAAS na literatura ainda é escassa. Estudos ${ }^{(2,3,23)}$ que buscaram avaliar crianças por meio do THAAS observaram que o instrumento mostrou-se eficiente e altamente sugestivo na detecção de alterações da atenção sustentada.

Pesquisadores $^{(24,25)}$ têm buscado evidenciar a ligação entre PAC e habilidade atencional de crianças. Estudo ${ }^{(24)}$ que utilizou o ACPT para avaliar a atenção de indivíduos com TDAH pré e pós-medicação demonstrou a melhora nos testes após utilização do psicoestimulante, fato que concorda com a presente pesquisa. Outro estudo ${ }^{(25)}$ evidenciou que a relação entre a desatenção e o TPAC não é direta, mas que ambos podem estar relacionados, já que a probabilidade de alteração do TPAC aumenta quando associada à desatenção.

Na presente pesquisa, verificou-se que, sem o uso da medicação, cinco crianças apresentaram alteração em algum item do THAAS. Após o uso, apenas três permaneceram com algum item alterado. Tillery et al. ${ }^{(11)}$ utilizaram o ACPT para analisar a atenção sustentada e testes do PAC em 32 crianças com TDAH pré e pós-medicação e verificaram que, nos testes do PAC, não houve melhora do desempenho após uso do medicamento. No entanto, constatou-se melhora no desempenho da atenção/impulsividade no teste $\mathrm{ACPT}^{(11)}$. Desta forma, revelou-se que o medicamento parece atuar na atenção auditiva sustentada, reduzindo os sintomas de impulsividade e de desatenção, conforme resultados do teste $\mathrm{ACPT}^{(11)}$. Tal fato também confirma a presente pesquisa quanto aos resultados encontrados do THAAS no quesito desatenção, em que se observou melhora significativa do desempenho dos pacientes após o uso da medicação.

Cabe ressaltar que, no presente estudo, todas as crianças avaliadas eram do gênero masculino, que, segundo pesquisadores, geralmente desenvolve de forma mais tardia o controle dos impulsos, apresentando mais sintomas de impulsividade ${ }^{(26)}$. Outro 
fator que deve ser levado em consideração é que essas crianças tinham entre 8 e 10 anos de idade e que o fator idade pode influenciar no quesito impulsividade. Pesquisa ${ }^{(6)}$ observou que, quanto mais nova a criança, maior a tendência a ser impulsiva.

Em relação ao item pontuação total de erros, por conseguinte, também houve melhora no desempenho, pois este item é formado pela soma dos erros de desatenção com os erros de impulsividade. Assim, a melhora do item desatenção influenciou diretamente a melhora da pontuação total de erros.

Quanto ao quesito vigilância, os resultados não demonstraram variações significativas, sinalizando que os indivíduos não apresentaram diferença no desempenho entre o início e o final do teste, antes e após o uso da medicação. Pesquisadores ${ }^{(6)}$ apontaram que o decréscimo da vigilância pode aparecer em condições que exijam um nível atencional por um tempo continuado e que um pequeno decréscimo é considerado normal. Contudo, quando os erros começam a aparecer por conta do decréscimo de vigilância, as crianças tendem a ficar ansiosas, repercutindo na piora de seu desempenho. Além disso, os erros de impulsividade podem aparecer devido à duração longa do teste.

No presente estudo, a média do decréscimo de vigilância foi de 2,25 antes e, após o uso da medicação, de 1,87, aproximando-se do padrão de normalidade encontrado por Feniman ${ }^{(6)}$, que é de 1,5.

Observou-se, também, que a maioria das crianças apresentou alterações de fala, bem como alterações na atenção sustentada. Moraes et al. ${ }^{(5)}$ confirmam os achados desta pesquisa, mostrando que a presença de alterações na habilidade de atenção auditiva aumenta a probabilidade de alterações na fala, em consequência da maior dificuldade desses sujeitos em lidar com os estímulos sonoros.

Os estudos que utilizaram o THAAS revelaram que, quando alterados os aspectos de vigilância e atenção sustentada, pode-se apontar para um prejuízo de concentração, que pode interferir na aprendizagem ${ }^{(6)}$, como também indica o presente estudo. Assim, a habilidade atencional auditiva está relacionada intrinsecamente ao desempenho, à aquisição e à consolidação da aprendizagem, funcionando como preditor essencial e requisito básico para a consecução e domínio dos aspectos acústicos e fonéticos das estruturas linguísticas, principalmente no período de alfabetização ${ }^{(2)}$. Portanto, o emprego do THAAS pode contribuir e muito nesses casos, considerando a assertiva de que os maiores prejuízos escolares das crianças, atualmente, decorrem da desatenção $0^{(3)}$.

Também se pode constatar que o THAAS viabiliza observar como cada sujeito se comporta e lida com estímulos relevantes e irrelevantes em seu ambiente. A medicação parece influenciar na melhora do foco atencional, mostrando que, após sua administração, o desempenho das crianças em atentar-se e concentrar-se em uma tarefa por um período de tempo foi melhor, levando-se em conta a ação intencional na direção de um estímulo, a inibição e a resistência à distração ${ }^{(5)}$. Este resultado confirma outros estudos ${ }^{(11,14)}$ que indicaram a melhora da atenção mediante a estimulação do SNC pelo uso de medicação. Contudo, deve-se levar em consideração a possibilidade de ter ocorrido o efeito de aprendizagem, devido ao tempo entre as testagens ter sido relativamente curto.

O tratamento por meio de medicamentos farmacológicos na população com TDAH vem crescendo ano após ano, sendo o metilfenidato o psicoestimulante mais consumido no mundo. A substância age como um estimulante do SNC, aumentando a disponibilidade dos neurotransmissores dopamina e noripinefrina na fenda sináptica. Durante o uso da medicação, os efeitos esperados são diminuição da inquietação motora e aumento de concentração, atenção e memória ${ }^{(14)}$. Além disso, espera-se a melhora do desempenho em testes de PAC em crianças com TDAH, após o uso do medicamento ${ }^{(14)}$.

Reforça-se que a medicalização não é o único meio de intervenção possível e que, em virtude do alto potencial para abuso e dependência de substâncias psicoestimulantes, faz-se necessária a reflexão sobre o uso desses medicamentos, que devem ser prescritos com cautela ${ }^{(14,27)}$. Além disso, o medicamento pode modificar condutas em algumas situações ${ }^{(14)}$. Por esses motivos, sua utilização é mais indicada nos casos em que se evidencia baixo rendimento escolar e/ou problemas para relação interpessoal ${ }^{(1)}$.

Assim sendo, é bastante pertinente no contexto clínico e escolar a determinação diagnóstica dos mecanismos atencionais por meio de instrumentos que avaliem o desempenho atencional $^{(23)}$. Quando presente a alteração nessa habilidade, o enfoque deverá ser no treinamento da atenção. No entanto, quando a impulsividade for a causadora da desatenção, a criança precisará, primeiramente, aprender a controlar o tempo de respostas, antes de treinar a atenção ${ }^{(28)}$.

Algumas limitações deste estudo devem ser mencionadas, como a dificuldade em agrupar pacientes com diagnóstico de TDAH sem outras comorbidades atreladas. Além disso, ocorreram problemas com o abastecimento da medicação Ritalina Comum e Ritalina LA nas farmácias, no período de coleta de dados, fazendo com que muitas crianças ficassem sem a medicação, impactando, assim, o número de pacientes da amostra, que ficou reduzido.

Apesar dessas dificuldades, o estudo contribui para a reflexão de que é importante a avaliação das habilidades multimodais, como a atenção, especialmente em indivíduos com TDAH. Desse modo, pode-se quantificar o benefício da medicação no quesito atenção, podendo contribuir também para a implantação de outras formas de tratamento. Cacace e McFarland ${ }^{(29)}$ recomendaram que, na bateria dos testes de PAC, sejam inseridas avaliações multimodais.

A relevância deste estudo consistiu em mostrar os efeitos da medicação na atenção sustentada de crianças com diagnóstico de TDAH. Acredita-se que a medicação psicoestimulante, quando corretamente indicada, auxilia o fonoaudiólogo no diagnóstico preciso na avaliação do PAC, uma vez que alterações comportamentais são menos evidentes e habilidades auditivas podem ser avaliadas de forma mais fidedigna. Sugerem-se mais pesquisas que avaliem a correlação entre PAC e TDAH para auxiliar o processo de reorganização do indivíduo, melhorando sua qualidade de vida.

Apesar dos poucos estudos na literatura com THAAS, é de extrema importância que mais pesquisas utilizem esse instrumento para avaliação da atenção sustentada, a fim de contribuir com ferramentas que sejam mais apropriadas e sensíveis para um diagnóstico diferencial e para a delimitação do seu emprego clínico.

\section{CONCLUSÃO}

Houve melhora no desempenho do teste que avaliou a habilidade atencional auditiva após o uso da medicação psicoestimulante, com destaque para o item desatenção do teste THAAS. 


\section{AGRADECIMENTOS}

Agradecemos aos pacientes e equipe do núcleo Desenvolver do Hospital Universitário Professor Polydoro Ernani de São Thiago da UFSC.

\section{REFERÊNCIAS}

1. Rotta NT, Ohlweiller L, Riesgo RS, editores. Transtornos da aprendizagem: abordagem neurobiológica e multidisciplinar. 2. ed. Porto Alegre: Art Med; 2016.

2. Lemos ICC, Feniman MR. Teste de Habilidade de Atenção Auditiva Sustentada (THAAS) em crianças de sete anos com fisssura labiopalatina. Rev Bras Otorrinolaringol. 2010;76(2):199-205.

3. Picolini MM, Stivanin D, Sampaio AR, Salvador KK, Lauris JRP, Feniman MR. Auditory attention: time of day and type of school. Int Arch Otorhinolaryngol. 2014;14(2):174-9.

4. Mondelli MFCG, Carvalho FRP, Feniman MR, Lauris JRP. Perda auditiva leve: desempenho no Teste da Habilidade de Atenção Auditiva Sustentada. Pró-fono Rev de Atualização Científica. 2010;22(3):24550. http://dx.doi.org/10.1590/S0104-56872010000300015.

5. Moraes TFD, Maximino LP, Feniman MR. A habilidade de atenção auditiva sustentada em crianças com fissura labiopalatina e transtorno fonológico. Rev Soc Bras Fonoaudiol. 2011;16(4):436-40. http:// dx.doi.org/10.1590/S1516-80342011000400012.

6. Feniman MR, Lemos ICC. A habilidade de atenção auditiva sustentada em crianças. Rev Bras Otorrinolaringol. 2007;25(4):280-4.

7. Carvalho NG, Novelli CVL, Colella-Santos MF. Fatores na infância e adolescência que podem influenciar o processamento auditivo: revisão sistemática. Rev CEFAC. 2015;17(5):1590-03. http://dx.doi. org/10.1590/1982-0216201517519014.

8. Ramos BD. But, after all, why is it important to assess the auditory processing? Rev Bras Otorrinolaringol. 2013;79(5):529-29. http:// dx.doi.org/10.5935/1808-8694.20130097. PMid:24141665.

9. Iliadou V, Ptok M, Grech H, Pedersen E, Brechmann A, Deggouj $\mathrm{N}$, et al. A European perspective on auditory processing disorder-current knowledge and future research focus. Front Neurol. 2017;8(21):622. http://dx.doi.org/10.3389/fneur.2017.00622. PMid:29209272.

10. Cunha VLO, Silva C, Lourencetti MD, Padula NAMR, Capellini SA. Desempenho de escolares com transtorno de déficit de atenção e hiperatividade em tarefas metalinguísticas e de leitura. Rev CEFAC. 2013;15(1):40-50. http://dx.doi.org/10.1590/S1516-18462012005000003.

11. Tillery KL, Katz J, Keller WD. Effects of Methylphenidate (Ritalin) on auditory performance in children with attention and auditory processing disorders. J Speech Lang Hear Res. 2000;43(4):893-901. http://dx.doi.org/10.1044/jslhr.4304.893. PMid:11386476.

12. Cavadas M, Pereira LD, Mattos P. Efeito do metilfenidato no processamento auditivo em crianças e adolescentes com transtorno do déficit de atenção/hiperatividade. Arq Neuropsiquiatr. 2007;65(1):138-43. http:// dx.doi.org/10.1590/S0004-282X2007000100028. PMid:17420844.

13. Abdo AGR, Murphy CFB, Schochat E. Habilidades auditivas em crianças com dislexia e transtorno do déficit de atenção e hiperatividade. Pró-fono Rev de Atualização Científica. 2010;22(1):25-30. http:// dx.doi.org/10.1590/S0104-56872010000100006.
14. Itaborahy C, Ortega F. O metilfenidato no Brasil: uma década de publicações. Cien Saude Colet. 2013;18(3):803-16. http://dx.doi. org/10.1590/S1413-81232013000300026. PMid:23546207.

15. ABA: Associação Brasileira de Audiologia. Fórum: diagnóstico audiológico - 2016. In: Anais do $31^{\circ}$ Encontro Internacional de Audiologia; 2016; São Paulo. São Paulo: ABA; 2016.

16. Alda JA, Serrano-Troncoso E. Attention-Deficit Hyperactivity Disorder: Agreement between Clinical Impression and the SNAP-IV Screening Tool. Actas Esp Psiquiatr. 2013;41(2):76-83. PMid:23592067.

17. Nunes CL, Pereira LD, Carvalho GS. Scale of Auditory Behaviors e testes auditivos comportamentais para avaliação do processamento auditivo em crianças falantes do português europeu. CoDAS. 2013;25(3):209-15. http://dx.doi.org/10.1590/S2317-17822013000300004. PMid:24408330.

18. USP: Universidade de São Paulo. Teste de Habilidade de Atenção Auditiva Sustentada (THAAS) [Internet]. Bauru: Faculdade de Odontologia de Bauru; 2020 [citado em 2020 Jul 14]. Disponível em: http://www.thaas.fob.usp.br/

19. Prando ML, Jacobsen GM, Moraes AL, Gonçalves HA, Fonseca RP. Avaliação da linguagem e do processamento auditivo na caracterização neuropsicológica do TDAH: revisão Sistemática. Psicol Pesqui. 2013;7(1):23-36. http://dx.doi.org/10.5327/Z1982-1247201300010004.

20. Miranda AR, Bruera JA, Serra SV. Scale of auditory behaviors: normative reference values for healthy argentinian children. Acta Neuropsychol. 2016;15(2):119-26. http://dx.doi.org/10.5604/01.3001.0010.2403.

21. Marcon GTG, Sardagna HV, Schussler D. O questionário SNAP-IV como auxiliar psicopedagógico no diagnóstico preliminar do Transtorno de déficit de atenção e hiperatividade (TDAH). Constr Psicopedag. 2016;25(24):99-118.

22. Fostick L. The effect of attention-deficit/hyperactivity disorder and methylphenidate treatment on the adult auditory temporal order judgment threshold. J Speech Lang Hear Res. 2017;60(7):2124-8. http://dx.doi.org/10.1044/2017_JSLHR-H-16-0074. PMid:28672285.

23. Rissatto A, Lauris J, Mondelli M, Feniman M. Applicability of the free field Sustained Auditory Attention Ability Test (SAAAT). Int Arch Otorhinolaryngol. 2012;16(2):269-77. http://dx.doi.org/10.7162/ S1809-97772012000200017. PMid:25991945.

24. Keith RW, Engineer P. Effects of methylphenidate on the auditory processing abilities of children with attention deficithyperactivity disorder. J Learn Disabil. 1991;24(10):630-6. http://dx.doi. org/10.1177/002221949102401006. PMid:1783870.

25. Gyldenkaerne P, Dillon H, Sharma M, Purdy SC. Attend to this: the relationship between auditory processing disorders and attention deficits. J Am Acad Audiol. 2014;25(7):676-87, quiz 706-7. http:// dx.doi.org/10.3766/jaaa.25.7.6. PMid:25365370.

26. Groot AS, Sonneville LMJ, Stins JF, Boomsma DI. Familial influences on sustained attention and inhibition in preschoolers. J Child Psychol Psychiatry. 2004;45(2):306-14. http://dx.doi.org/10.1111/j.14697610.2004.00222.x. PMid:14982244.

27. Storebø O, Ramstad E, Krogh H, Nilausen T, Skoog M, Holmskov $\mathrm{M}$, et al. Methylphenidate for children and adolescents with attention deficit hyperactivity disorder (ADHD). Cochrane Database Syst Rev. 2015;(11):CD009885. http://dx.doi.org/10.1002/14651858.CD009885. pub2. PMid:26599576.

28. Ribeiro SP. TCC e as funções executivas em crianças com TDAH Rev Bras Ter Cogn. 2016;12(2):126-34.

29. Cacace AT, McFarland DJ. Factors influencing tests of auditory processing: a perspective on current issues and relevant concerns. J Am Acad Audiol. 2013;24(7):572-89. http://dx.doi.org/10.3766/ jaaa.24.7.6. PMid:24047945. 